\title{
Prenatal Diagnosis of Intracranial Tumors and the Difficulties in Prognostication: A Report of Three Cases
}

\author{
${ }^{1}$ Akhila Vasudeva, ${ }^{2}$ Sapna V Amin, ${ }^{3}$ Rajagopal Kadavigere, ${ }^{4}$ Katta M Girisha, ${ }^{5}$ Lavanya Rai
}

\begin{abstract}
Prenatal diagnosis of intracranial tumors generally implies a poor prognosis. We present three such cases, where prognostication was difficult. We attempted to correlate our prenatal counseling with postnatal follow-up/postabortal diagnosis. In the first case, tumor was diagnosed at 37 weeks. Ultrasound and fetal/neonatal MRI suggested a malignant intraventricular tumor. Anticipating guarded prognosis, parents refused neurosurgical intervention. At 1 year, child has normal neurodevelopment. Further magnetic resonance imagings (MRIs) show tumor shrinkage, pointing to a benign tumor. In case two, a choroid plexus tumor was diagnosed at midtrimester anomaly scan. Since it was difficult to rule out a malignant tumor, pregnancy was terminated. However, MRI, autopsy, and histopathology confirmed a choroid plexus papilloma, which is known to have good prognosis. In case three, prenatal MRI showed features of neurological involvement in tuberous sclerosis. However child has no neurological manifestations at 1 year of age. Hence, prognostication of prenatally diagnosed brain tumors remains a challenge.
\end{abstract}

Keywords: Choroid plexus papilloma, Congenital brain tumor, Fetal intracranial tumor, Fetal MRI, Prenatal diagnosis, Prognosis of congenital brain tumor.

How to cite this article: Vasudeva A, Amin SV, Kadavigere R, Girisha KM, Rai L. Prenatal Diagnosis of Intracranial Tumors and the Difficulties in Prognostication: A Report of Three Cases. Int J Infertil Fetal Med 2015;6(2):88-91.

Source of support: Nil

Conflict of interest: None

Date of received: $05-05-15$

Date of acceptance: 15-06-15

Date of publication: August 2015

\section{INTRODUCTION}

Intracranial tumors presenting in prenatal life pose several challenges while managing the pregnancy.

\footnotetext{
${ }^{1,2}$ Associate Professor, ${ }^{3-5}$ Professor and Head

1,2,5 Department of Obstetrics and Gynecology, Kasturba Medical College, Manipal University, Manipal, Karnataka, India

${ }^{3}$ Department of Radiology, Kasturba Medical College, Manipal University, Manipal, Karnataka, India

${ }^{4}$ Department of Medical Genetics, Kasturba Medical College Manipal University, Manipal, Karnataka, India
}

Corresponding Author: Akhila Vasudeva, Associate Professor Department of Obstetrics and Gynecology, Kasturba Medical College, Manipal University, Manipal, Karnataka, India, Phone: 09448931690, e-mail: akhilavasudeva@gmail.com
We present three such cases, which proved to be difficult in terms of prognostication.

\section{CASE REPORTS}

\section{Case 1}

Tumor was diagnosed at 37 weeks of gestation. Ultrasound showed severe unilateral ventriculomegaly, and thinned out cerebral mantle (Fig. 1A). Fetal MRI (Fig. 1B) showed a well defined lobulated mass measuring $4.1 \times 3.8 \mathrm{~cm}$ along the anterior falx, extending into left cerebral hemisphere. An intracranial neoplasm was suspected. Dismal prognosis was conveyed to the parents. Baby was not vigorous at birth, developed multiple episodes of multifocal clonic seizures on day. ${ }^{1}$ Electrolyte abnormalities were corrected and baby was started on anticonvulsants.

Neonatal magnetic resonance imaging (MRI) (Fig. 1C) revealed a large intraventricular tumor in the left lateral ventricle, infiltrating into adjacent brain parenchyma. Gross hydrocephalus was seen. Possibility of a primitive neuroectodermal tumor (PNET) or a choroid plexus carcinoma was considered. Neurosurgeons advised ventriculoperitoneal shunt, and planned tumor removal later. However, after being thoroughly counseled regarding the guarded neurological outcome, parents were not keen on further treatment hence, baby was discharged at request. A repeat of MRI at 6 months of age (Fig. 1D) showed significant reduction in the cystic component of tumor, although solid part remained the same. Magnetic resonance imaging at 1 year (Fig. 1E) did not show any interval change in the mass or ventriculomegaly. Child is currently one and a half years of age. Neurodevelopmental milestones are appropriate for age, although there was an initial delay in achieving milestones. There is roving nystagmus, vision is normal, deep tendon reflexes are exaggerated. Overall, child appears to be doing well. There is no increase in head size and no features of raised intracranial pressure, hence neurosurgeons advised regular follow-ups with continued anticonvulsants.

\section{Case 2}

There was bilateral cerebral ventriculomegaly at the 19th week ultrasound, and multiple choroid plexus cysts. About $2 \mathrm{~cm}$ of choroid plexus on the right appeared enlarged, echogenic, leading onto midline shift (Fig. 2A). 
Choroid plexus papilloma was suspected. Amniocentesis showed normal karyotype. Uncertainty on prognosis lead the parents to opt for pregnancy termination . Post abortal fetal MRI (Fig. 2B) showed an intraventricular lesion measuring $3 \times 2.6 \mathrm{~cm}$ involving right lateral ventricle. Autopsy features (Fig. 2C), were suggestive of choroid
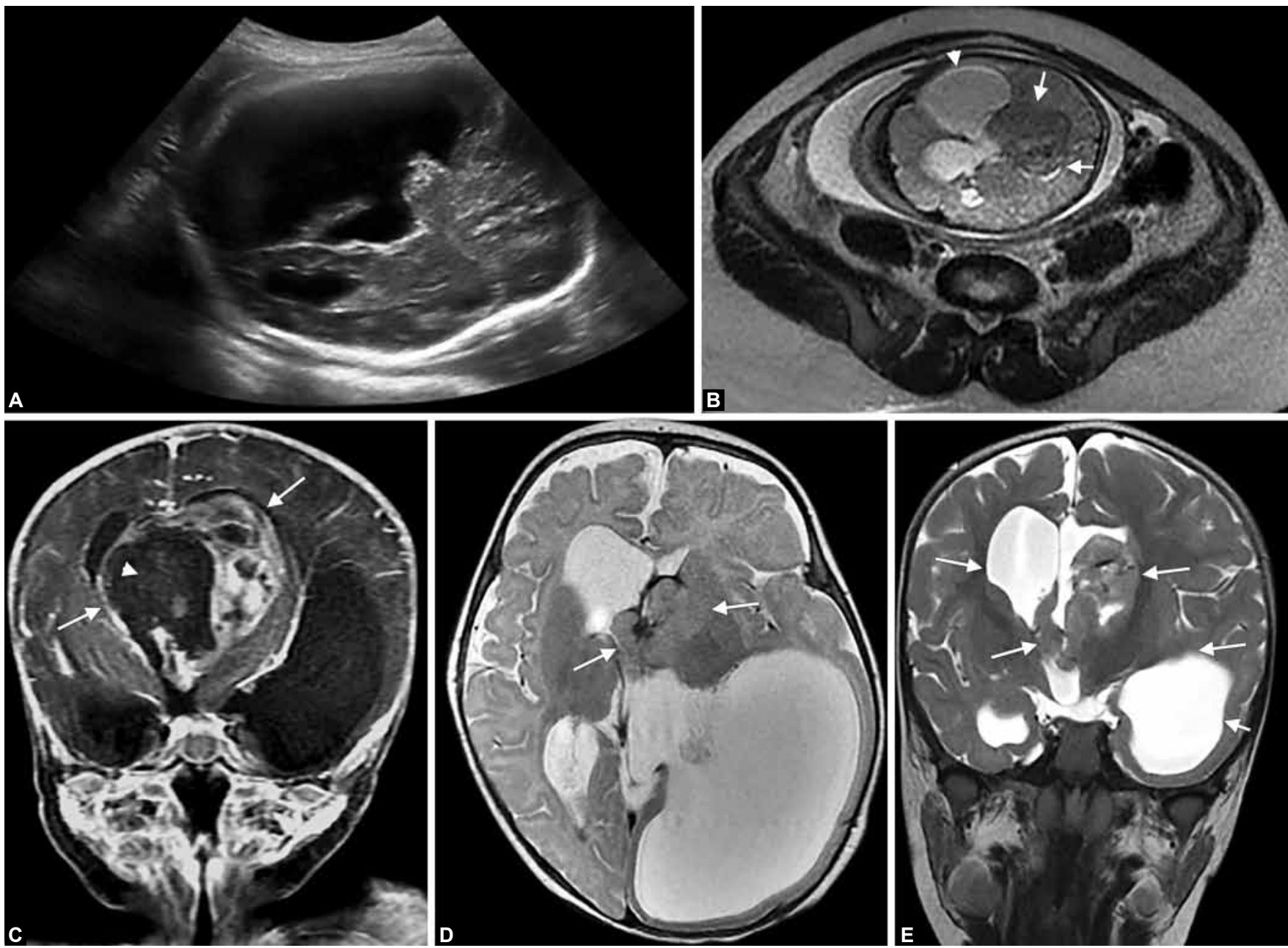

Figs 1A to E: (A) USG at 37 weeks showing severe unilateral ventriculomegaly with gross midline shift and thinning of cerebral mantle, (B) fetal MRI performed at 37 weeks of gestation shows a lobulated solid mass lesion at the level of foramen of Munro (arrows) causing asymmetric dilatation of the lateral ventricles (arrowhead), (C) postnatal MRI of the brain shows a large mass at the level of foramen of Munro (arrows) with large necrotic component (arrowhead) and large lateral ventricles $(L>R)$, (D) follow-up MRI after 6 months shows significant reduction in the size of the lesion, however, solid portion of the mass remaining same (arrows), (E) follow-up MRI after 12 months did not reveal any significant interval change compared to previous MRI performed at 6 months (long arrows) with persistence of lateral ventricular dilatation (small arrow)
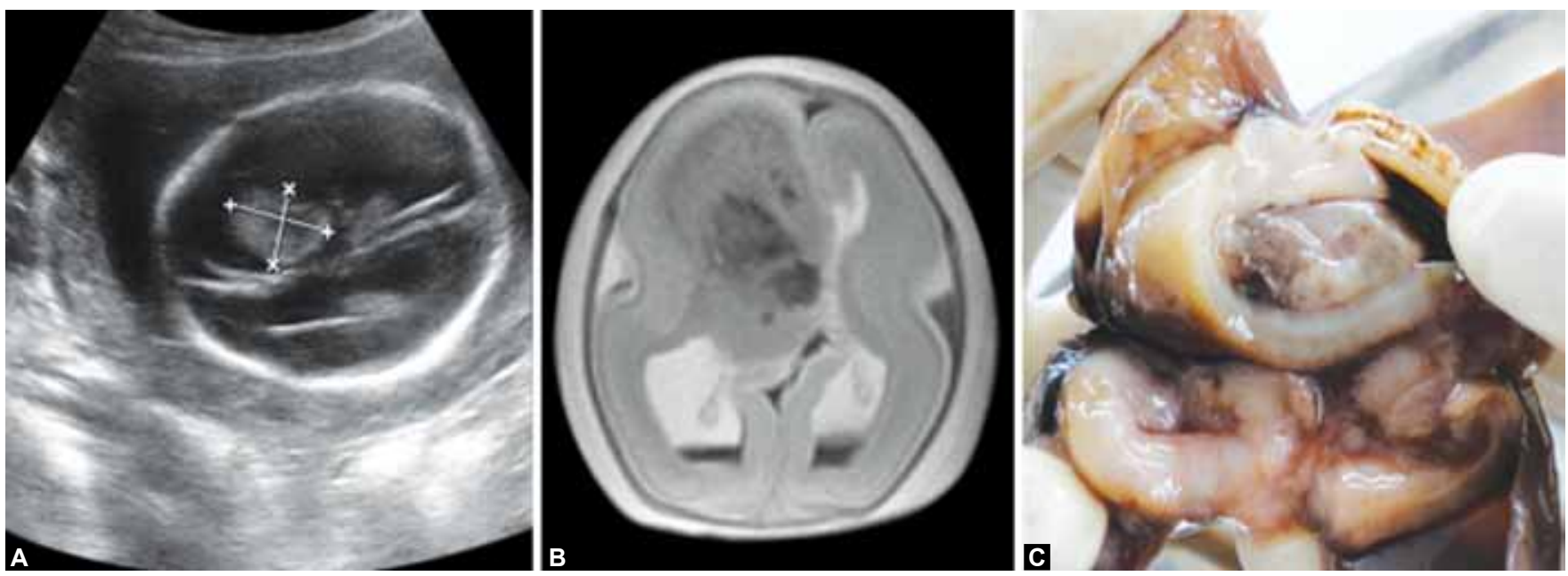

Figs 2A to C: Choroid plexus papilloma: (A) USG image suspicious of choroid plexus tumor causing midline shift, (B) MRI of the abortus showing tumorous lesion in the choroid plexus and $(C)$ fetal autopsy showing the papilloma choroid plexus 
plexus papilloma/carcinoma. Histopathology confirmed a choroid plexus papilloma.

\section{Case 3}

Multiple fetal intracardiac rhabdomyomata, were diagnosed at 22 weeks of pregnancy. Fetal MRI done at 27 weeks of gestation, suggested bilateral subependymal nodules, which were not seen in antenatal neurosonograms. Guarded neurological prognosis was explained. Baby had typical Ash Leaf lesions, and multiple cardiac rhabdomyomata. Newborn MRI (Figs 3A to C) showed features consistent with tuberous sclerosis, in the form of multiple subependymal nodules, and radial white matter bands in both frontal lobes. Baby was started on prophylactic phenytoin and advised on regular followups. Possible effects on neurological prognosis explained to the couple. Currently, baby is one and a half year old. Rhabdomyomata have regressed. Neurodevelopment and other milestones have been normal so far. There have been convulsion.

\section{DISCUSSION}

Fetal intracranial tumors are rare encounters in general obstetric practice. Diagnosis may be missed in the ultrasound. Fetal MRI is very useful adjunct in the diagnosis. However, prognostication is difficult. As in case one, overall impression of fetal and neonatal MRI was that of a malignant tumor. Antenatal counseling on guarded neurological prognosis, led the couple take a decision against neurosurgical intervention. Severe neurodevelopmental sequel was anticipated in this child. However, normal neurodevelopment and follow-up MRIs are more suggestive of choroid plexus papilloma. Hence, prenatal prognostication proved to be difficult.
In case two, couple took an early decision on medical termination of pregnancy (MTP). It is hard to differentiate choroid plexus papilloma from carcinoma from prenatal imaging. ${ }^{1}$ Choroid plexus papilloma (as in this case) can be surgically resected and can have good prognosis, although there is a risk of malignant transformation. However, in a country, like India where legally MTP can be performed only up to 20 weeks, uncertain neurological prognosis will have to be conveyed early on. It was difficult to rule out choroid plexus carcinoma based on midtrimester scan. Again, diagnosis has helped but prognostication proved to be difficult.

In tuberous sclerosis ${ }^{2}$ brain lesions are cortical tubers and multiple subependymal hamartomas/nodules. In a prospective follow-up of five fetuses with cardiac rhabdomyomas and tuberous sclerosis ${ }^{3}$ two fetuses showed cerebral lesions in MRI which correlated well with clinical manifestations (severe epilepsy) on follow-up. In a retrospective review of fetuses with cardiac rhabdomyomas ${ }^{4}$ $49 \%$ had cerebral lesions in MRI. Majority of this group opted MTP. In general, fetal MRI is strongly recommended in order to anticipate childhood neurological manifestations. ${ }^{3}$ Cerebral lesions are responsible for the most severe manifestation of the disease, like seizures, mental retardation, behavioral abnormality. However, absence of cerebral lesions on MRI does not exclude neurodevelopmental sequel, as lesions may develop progressively over time. Clinical correlation is not perfect. In our case, prenatal detection of lesions prompted us to give guarded neurological prognosis. However, child so far has normal neurodevelopment.

Ventriculomegaly is a common manifestation of fetal brain tumor. ${ }^{5-7}$ In developing countries; fetal MRI is now beginning to be used in cases of ventriculomegaly detected
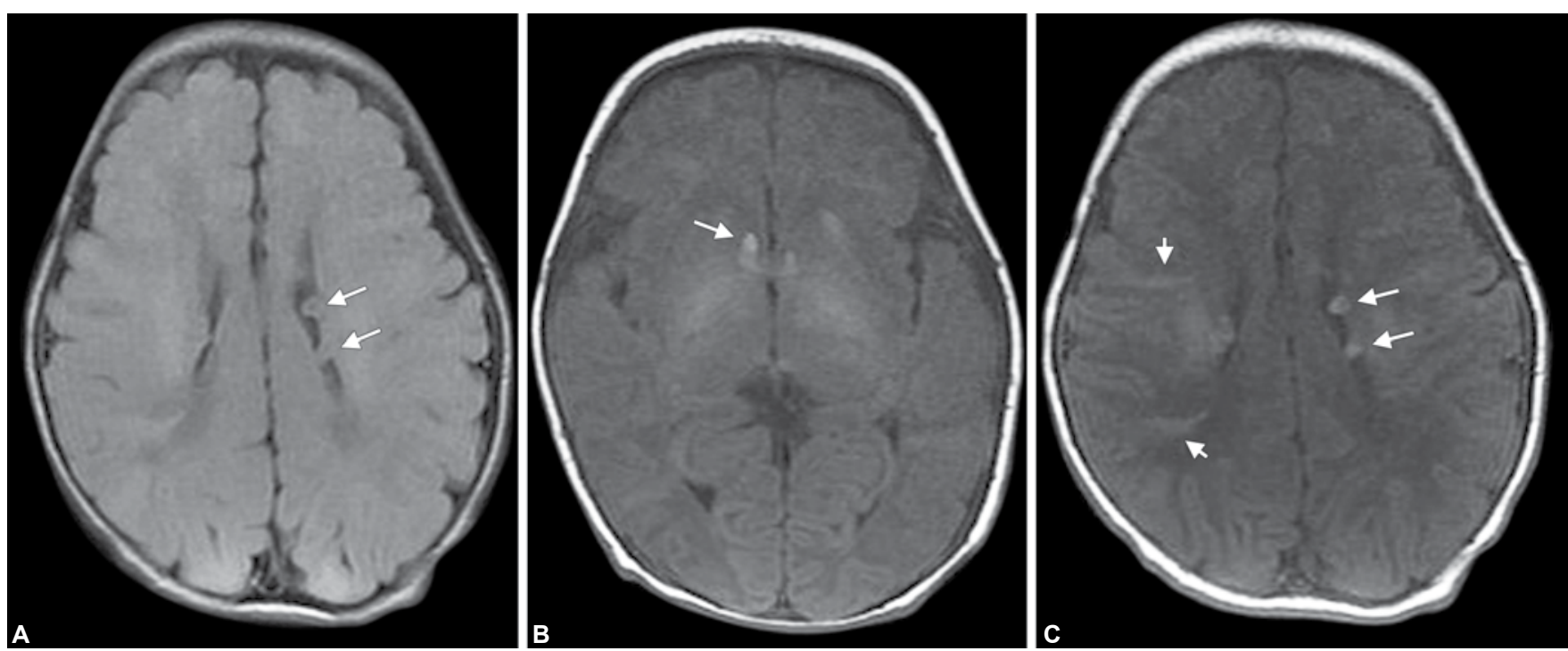

Figs 3A to C: Neonatal MRI showing features of tuberous sclerosis (A) multiple, well defined subependymal nodules hypointense on FLAIR sequence (arrow) (B and C) showing contrast enhancement along both lateral ventricles, (arrow) and (C) Radial white matter bands are also noted (small arrow). Magnetic resonance features are consistent with tuberous sclerosis 
in later half of gestation. There is a need to increase use of MRI and also to train more radiologists/obstetricians in fetal MRI interpretation. Despite use of fetal MRI, definitive diagnosis of individual tumor type is difficult, and accurate prognostication is challenging. ${ }^{1}$ Certain tumor types can be accurately diagnosed by prenatal MRI example: lipoma, mature terratoma; but not all. In terms of tumor type, those carrying good prognosis are choroid plexus papilloma, glioma, lipoma and astrocytoma of low degree; whereas tumors, like teratoma, and primitive neurectodermal tumors are known to carry poor prognosis. ${ }^{8,9}$

However, overall poor prognosis can be conveyed. In a literature review of 154 cases of fetal brain tumors, survival was only $15 \% .{ }^{10}$ However, tumors can be resected successfully if they are small and favorably located, as in choroid plexus papilloma. ${ }^{5}$ Generally, diagnosis before the third trimester implies dismal prognosis. If there is a complex mass with distorted cranial anatomy, poor survival can be conveyed. On the other hand, well delineated echogenic masses in corpus callosum/choroid plexus, are likely to be lipomas, and intact survival is highly likely. ${ }^{6}$ Other imaging features suggestive of malignancy, hence poor prognosis are ill-defined margins, perifocal edema, cerebrospinal fluid metastasis, and lack of cystic/calcified areas. ${ }^{11}$

Fetal intracranial tumors are usually isolated abnormalities. ${ }^{12}$ Magnetic resonance imaging can be especially helpful in identifying exact location of tumor, local extension, and the effects on adjacent brain structure, all of which are important in prognostication. ${ }^{12}$ This evaluation is also helpful in counseling about the results of surgery.

Although prenatal imaging has got limitations in prognostication, it gives valuable information for those neonates who are sick, require life support and are thus difficult candidates for postnatal imaging.

\section{CONCLUSION}

Fetal brain tumors are rare. Prenatal MRI is very helpful in diagnosis. However, prognostication is difficult and prenatal counseling on future neurological prognosis may prove challenging.

\section{ACKNOWLEDGMENT}

The work was partially supported by the grant from Indian Council of Medical Research (BMS No. 54/5/2010).

\section{REFERENCES}

1. Anselem O, Mezzetta L, Grangé G, et al. Fetal tumors of the choroid plexus: is differential diagnosis between papilloma and carcinoma possible? Ultrasound Obstet Gynecol 2011; 38(2):229-232.

2. Colosi E, Russo C, Macaluso G, Musone R, Catalano C. Sonographic diagnosis of fetal cardiac rhabdomyomas and cerebral tubers: a case report of prenatal tuberous sclerosis. J Prenat Med 2013 Oct;7(4):51-55.

3. Mühler MR, Rake A, Schwabe M, Schmidt S, Kivelitz D, Chaoui R, Hamm B. Value of fetal cerebral MRI in sonographically proven cardiac rhabdomyoma. Pediatr Radiol 2007 May;37(5):467-474.

4. Saada J, Hadj Rabia S, Fermont L, Le Bidois J, Bernardes LS, Martinovic J, Sonigo P, Dumez Y, Bonnet D, Benachi A. Prenatal diagnosis of cardiac rhabdomyomas: incidence of associated cerebral lesions of tuberous sclerosis complex. Ultrasound Obstet Gynecol 2009 Aug;34(2):155-159.

5. Severino M, Schwartz ES, Thurnher MM, Rydland J, Nikas I, Rossi A. Congenital tumors of the central nervous system. Neuroradiol 2010;52(6):531-548.

6. Carletti A, Colleoni GG, Perolo A, et al. Prenatal diagnosis of cerebral lesions acquired in utero and with a late appearance. Prenat Diagn 2009;29(4):389-395.

7. Sahin FK, Koken G, Cosar E, Koken R, Sahin O, Gokden M. A prenatal diagnosed case of primitive neuroectodermal tumor. Fetal Diagn Ther 2008;23(4):267-270.

8. Isaacs H.I. Perinatal brain tumors: a review of 250 cases. Pediatr Neurol 2002;27(4):249-261.

9. Isaacs H.II. Perinatal brain tumors: a review of 250 cases. Pediatr Neurol 2002;27(5):333-342.

10. Isaacs H. Fetal brain tumors: a review of 154 cases. Am J Perinatol 2009 Jun;26(6):453-466.

11. Parmar HA, Pruthi S, Ibrahim M, Gandhi D. Imaging of congenital brain tumors. Semin ultrasound CT MR 2011 Dec;32(6):578-589.

12. Milani HJ, Araujo Júnior E, Cavalheiro S, Oliveira PS, Hisaba WJ, Barreto EQ, Barbosa MM, Nardozza LM, Moron AF. Fetal brain tumors: prenatal diagnosis by ultrasound and magnetic resonance imaging. World J Radiol 2015 Jan 28;7(1):17-21. 